\title{
Utilização de aerossolterapia no contexto da COVID-19*
}

\author{
Use of aerosol therapy in the context of COVID-19*
}

\begin{abstract}
ANDRADE, Flávio Maciel Dias de ; RIBEIRO, Daniel da Cunha ${ }^{2}$; MARTINS, Jocimar Avelar'; NOGUEIRA, Ingrid Correia ${ }^{4}$; em nome do Comitê COVID-19 da ASSOBRAFIR.
\end{abstract}

\section{Resumo}

Esse documento tem por objetivo fornecer informações que possam servir de base para utilização de aerossolterapia pelos fisioterapeutas no contexto da COVID-19. Qualquer procedimento que provoca o aumento do fluxo aéreo sobre a mucosa respiratória infectada apresenta risco de contaminação do ser humano, em virtude da liberação para o ambiente, de um aerossol potencialmente infectado. A terapia inalatória é frequentemente utilizada em pacientes internados nas unidades de terapia intensiva. Pela alta demanda e por suas particularidades, a ASSOBRAFIR recomenda que o adequado uso da aerossolterapia siga rigorosamente as orientações descritas neste documento, baseadas em literatura científica atualizada. Essa recomendação tem o objetivo de se atingir os efeitos clínicos pretendidos, mas garantindo a proteção dos profissionais de saúde e pacientes.

Palavras-chave: Fisioterapia; aerossolterapia; COVID-19.

\footnotetext{
* Revisado por membros do Comitê COVID-19 da ASSOBRAFIR, nomeado por meio do memorando No 003/2020. Esta publicação é uma atualização da Comunicação Oficial "Utilização de aerossolterapia no contexto da COVID-19", chancelada pelo Comitê COVID-19 da ASSOBRAFIR, originalmente escrita pelos mesmos autores e divulgada em 11/05/2020 no endereço eletrônico https://assobrafir.com.br/wp-content/uploads/2020/05/ASSOBRAFIR-COVID19 AEROSSOLTERAPIA_2020.05.11.pdf.

1 Universidade Católica de Pernambuco (UNICAP), Recife, Pernambuco, Brasil. Email: flaviomacielandrade@yahoo.com.br. FMDA - https://orcid.org/0000-0001-9571-6551

2 FISIOUNI - Grupo de Fisioterapia, Belo Horizonte, Minas Gerais, Brasil. DCR - https://orcid.org/0000-0001-8940-6186

${ }^{3}$ Faculdade Dinâmica do Vale do Piranga, Ponte Nova, Minas Gerais, Brasil. JAM - https://orcid.org/0000-0002-5023-3131

${ }^{4}$ Centro Universitário Christus, Fortaleza, Ceará, Brasil. ICN - https://orcid.org/0000-0002-8711-817X
} 


\section{Abstract}

This document aims to provide information to serve as the basis for the use of aerosol therapy by physiotherapists in the context of COVID-19. Any procedure that causes an increase in airflow over the infected respiratory tract poses a risk of contamination, due to the release to the environment of potentially infected aerosol. Inhalation therapy is often used in patients admitted to intensive care units (ICU). Due to the high demand and particularities within the ICU, ASSOBRAFIR recommends that the proper use of aerosol therapy strictly follows the guidelines described in this document, which are based on up-to-date scientific literature. This recommendation aims to reach the intended clinical effects, but ensuring the safety of health professionals and patients at the same time.

Keywords: Physiotherapy; aerosol therapy; COVID-19.

\section{Objetivo}

O objetivo do presente posicionamento é fornecer informações que possam servir de base para a utilização de aerossolterapia pelos fisioterapeutas no contexto da COVID-19.

\section{Contextualização}

\section{O que são e como são produzidos os aerossóis?}

Os aerossóis são produzidos quando uma corrente de ar se choca com a superfície de um líquido, gerando pequenas partículas em suspensão na interface ar-líquido. $O$ tamanho dessas partículas é inversamente proporcional a velocidade do ar (fluxo aéreo). Dessa forma, qualquer procedimento que provoca o aumento do fluxo aéreo sobre a mucosa respiratória infectada apresenta risco de contaminação para o ser humano, em virtude da liberação para o ambiente, de um aerossol potencialmente infectado ${ }^{1}$.

Um procedimento gerador de aerossol é definido como qualquer procedimento que induza a produção de aerossol de tamanhos variados. De acordo com o Guideline on the Management of Critically Ill Adults with Coronavirus Disease 2019 - COVID-19 da European Society of Intensive Care Medicine e Society of Critical Care Medicine, publicado em 20 de março de 2020, os procedimentos geradores de aerossol na unidade de terapia intensiva (UTI) incluem intubação traqueal, broncoscopia, aspiração traqueal com sistema aberto, ventilação manual antes da intubação, desconexão do paciente do ventilador, ventilação mecânica não invasiva (VNI), realização de traqueostomia, reanimação cardiopulmonar (RCP) e administração de nebulização a jato².

\section{Aerossolterapia: definição e formas de realização}

Frequentemente, a aerossolterapia tem sido utilizada no tratamento das afecções do trato respiratório, seja no ambiente domiciliar, ambulatorial ou hospitalar. Tal modalidade de administração medicamentosa por via inalatória visa a deposição direta de fármacos no interior do trato respiratório, com redução do tempo de resposta e de efeitos colaterais, quando comparados às outras formas de administração (intravenosa, intramuscular ou oral).1 Diversos métodos estão disponíveis para este fim, incluindo os nebulizadores a jato, ultrassônicos e por membrana vibrating mesh nebulizer VMN; nebulímetros dosimetrados (metered dose inhalers - MDI); e nebulímetros liofilizados (dry powder inhalers - DPI). Na prática, os mais comumente utilizados são os nebulizadores a jato e nebulímetros dosimetrados ${ }^{3}$. 
Nos nebulizadores a jato, o aerossol é gerado quando uma alta taxa de fluxo de gás (oxigênio, ar comprimido medicinal ou ar ambiente), obtida por meio do efeito Bernoulli, choca-se com uma interface líquida, produzindo a pulverização das partículas que a compõem. Por sua vez, os nebulímetros dosimetrados não dependem de fonte de gás ou alimentação elétrica para funcionar, consistindo em um reservatório pressurizado que contêm a droga em solução, que será pulverizada pela compressão gerada no interior da câmara ${ }^{1}$.

\section{Risco de contaminação}

Diversos recursos são propostos para melhora da ventilação e oxigenação de pacientes com formas leves e moderadas de insuficiência respiratória, mas sua utilização no contexto da COVID-19 exige a avaliação do risco de produção de aerossol e consequente contaminação de profissionais de saúde e outros pacientes. Vale ressaltar que a capacidade do dispositivo minimizar esse risco deve ser considerada durante seu processo de escolha.

Nesse cenário, nebulizadores a jato não devem ser utilizados nos cuidados destinados aos pacientes com COVID-19. A administração de aerossóis na forma de nebulização a jato para pacientes com suspeita, ou confirmação, da COVID-19 pode aumentar o risco de contaminação dos profissionais de saúde, cuidadores, familiares e outros pacientes. Segundo Hui et al. (2014), os nebulizadores a jato foram responsáveis pela disseminação da SARS (Síndrome Respiratória Aguda Severa) em uma enfermaria hospitalar Chinesa em $2003^{4}$.

De acordo com a European Society of Intensive Care Medicine a Society of Critical Care Medicine, um percentual considerável de profissionais de saúde é infectado nos seus postos de trabalho ${ }^{2}$.

Apesar de não oficiais, estatísticas advindas da China, Itália e Espanha relatam que cerca de 10 a $20 \%$ dos profissionais de saúde desses países foram contaminados durante a assistência prestada aos pacientes com COVID-19, especialmente durante a realização de procedimentos geradores de aerossóis.

Os nebulizadores a jato causam vazamento lateral do ar expirado e a distância máxima de dispersão aumenta com a piora da lesão pulmonar ${ }^{5,6}$ :

1. Pulmão normal (Complacência $=70 \mathrm{ml} / \mathrm{cmH}_{2} \mathrm{O}$; consumo de oxigênio $=200 \mathrm{ml} / \mathrm{min}$ )

1. distância de dispersão $=45 \mathrm{~cm}$

2. Lesão pulmonar leve $\left(\right.$ Complacência $=35 \mathrm{ml} / \mathrm{cmH}_{2} \mathrm{O}$; consumo de oxigênio $=300 \mathrm{ml} / \mathrm{min}$ ) 1. distância de dispersão $=54 \mathrm{~cm}$

3. Lesão pulmonar grave (Complacência $=10 \mathrm{ml} / \mathrm{cmH}_{2} \mathrm{O}$; consumo de oxigênio $=500 \mathrm{ml} /$ $\min$ )

1. distância de dispersão $=80 \mathrm{~cm}$.

A nebulização a jato é possivelmente o procedimento de maior risco para transmissão viral nosocomial e deve ser realizada apenas quando absolutamente necessária, em ambientes de pressão negativa, por pessoal altamente treinado e utilizando todos os equipamentos de proteção individual (EPIs) recomendados. Uma boa alternativa ao nebulizador a jato é o metered-dose inhaler (MDI). Este dispositivo tem capacidade para oferecer com eficiência os medicamentos aerolizados, com risco diminuído de dispersão de partículas?. 


\section{Aerossolterapia em pacientes sob ventilação mecânica}

Recomenda-se evitar a desconexão do circuito para administração de fármacos por via inalatória ${ }^{1,3,8}$.

1. Preferir o uso do MDI (também conhecido como “puff”) (Figura 1).

2. Dentre os dispositivos recomendados para realização do MDI, incluem-se: câmaras espaçadoras não colapsáveis, câmaras espaçadoras colapsáveis, conectores em linha, dispositivos HME (heat and moisture exchangers - HME; do inglês, trocadores de calor e umidade) e sistemas de aspiração fechado com conector para MDI. (Figuras 2, 3 e 4).

3. Na hipótese da não disponibilidade de fármacos tipo MDI, há possibilidade de nebulização com dispositivos ultrassônicos ou de malha vibratória, sendo este mais eficiente (Figura 5). Os mesmos devem ser conectados em linha com o circuito do ventilador mecânico, evitando sua desconexão e possível contaminação no ambiente.

4. Sob ventilação mecânica invasiva ou não invasiva, na hipótese de uso da umidificação ativa, para evitar depósito e desconexão do circuito ventilatório, sugere-se o uso do dispositivo MDI no ramo inspiratório, próximo ao "Y". Os nebulizadores ultrassônicos ou de malha vibratória podem ser acoplados diretamente à câmara umidificadora ou no ramo inspiratório, próximo ao ventilador mecânico.

5. Ao se utilizar a umidificação passiva (HME ou HMEF), na ventilação mecânica invasiva ou não invasiva, o dispositivo para aplicação do MDI ou nebulização (dispositivos ultrassônicos ou de malha vibratória, quando aplicáveis) deve ser posicionado entre o filtro e a via aérea artificial (Figura 1).

6. O uso do dispositivo para aplicação do MDI ou nebulização (quando aplicável) entre o filtro e a via aérea artificial deve seguir rigorosamente a indicação da seta contida no instrumento. A mesma deve estar apontada para o paciente, evitando assim o depósito de aerossol no filtro.

Para realização da aerossolterapia em pacientes sob ventilação mecânica não invasiva, as recomendações seguem as supracitadas, variando somente de acordo com o circuito e o tipo de umidificação utilizados:

1. Se a administração acontece em ventiladores de circuito duplo, manter as recomendações da ventilação invasiva.

2. Se a administração acontece em ventiladores de circuito único:

1. Na hipótese de uso de umidificação passiva, o dispositivo para aplicação de MDI ou nebulização deve ser posicionado entre a interface e o filtro.

2. Na hipótese de uso de umidificação ativa, o posicionamento do dispositivo deve ocorrer entre a porta expiratória em " $\mathrm{T}$ ” e a interface. 
Figura 1 | Cânister para administração de "puff” utilizando adaptador para metered-dose inhaler (MDI). O gerador de partículas inaladas deverá ser posicionado a $20-30 \mathrm{~cm}$ do tubo orotraqueal (TOT), entre o TOT e o Y do circuito e sua administração deve ser sincronizada com o início do fluxo inspiratório. HMEF: Heat and Moisture Exchanger Filter.

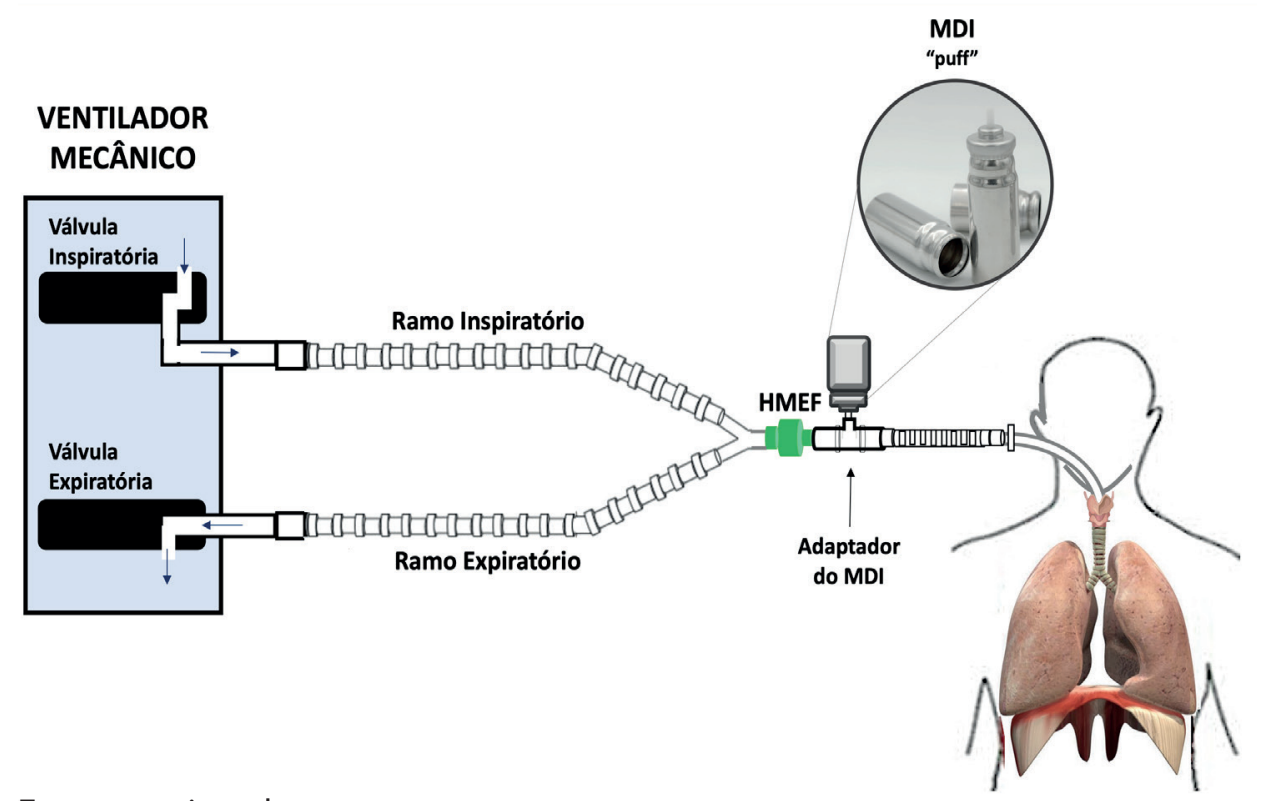

Fonte: arquivos dos autores.

Figura 2 | Adaptadores para utilização do metered-dose inhaler(MDI) durante a ventilação mecânica.

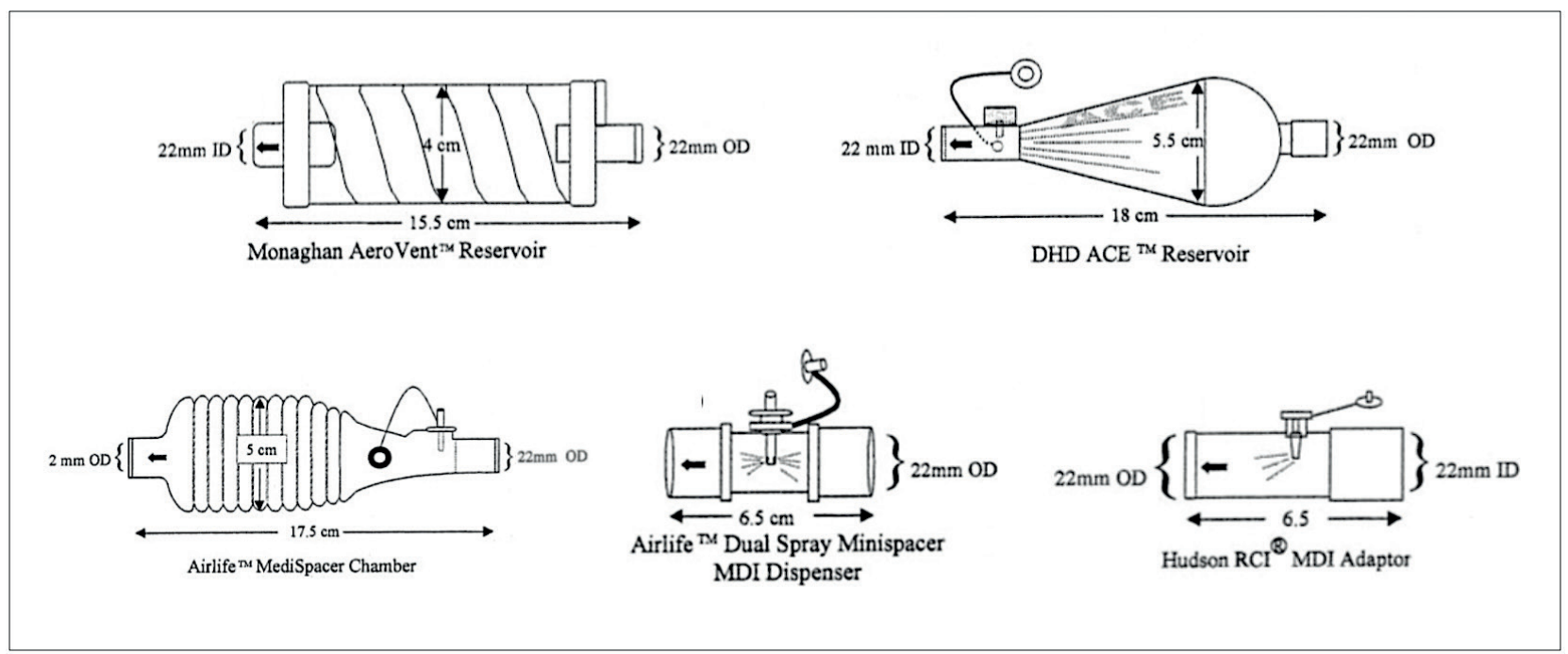

Fonte: Adaptada de Dhand, $2000^{9}$. 
Figura 3 | Trocadores de calor e umidade (Heat and moisture exchangers - HMEs) com entrada para administração de aerossolterapia durante a ventilação mecânica. A: CircuVent (Hudson RCI); B: Humid-Flo HME (Hudson RCI); C: AirLife (CareFusion).

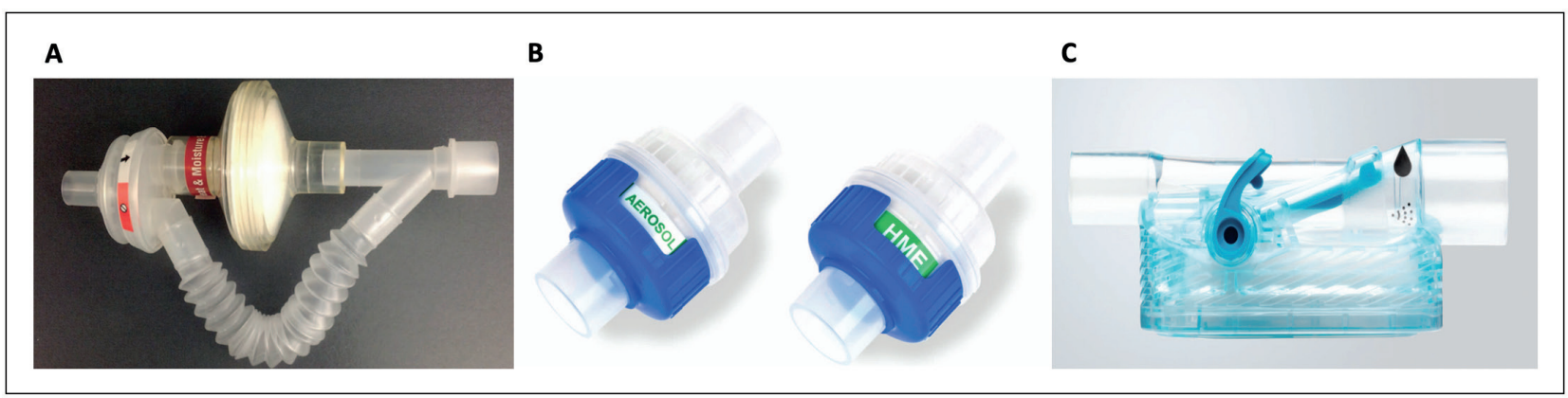

Fonte: Adaptada de Ari, 2015³.

Figura 4 | Sistema fechado de aspiração com adaptador para aerossolterapia.

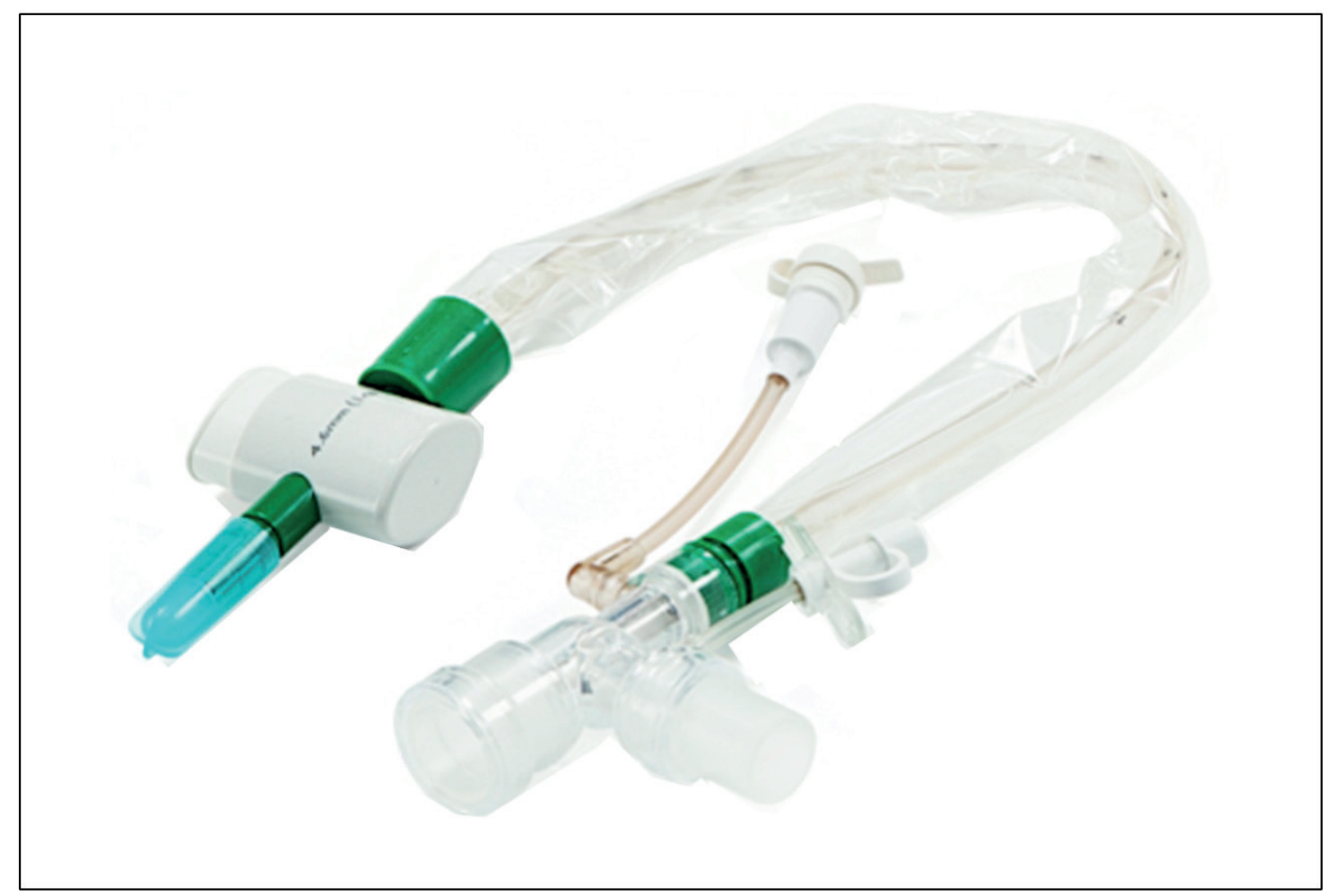

Fonte: Respiratory Therapeutics Group, $2020^{11}$. 
Figura 5 | Exemplo de um nebulizador com malha vibratória (MESH nebulizer), antes da sua administração com volume de $3 \mathrm{ml}$ de solução salina normal (seta 1) (A); após a cessação prematura da nebulização com volume residual de $0,4 \mathrm{ml}$ (seta 2) (B) e, após sua utilização com êxito, com o mínimo volume residual (C).
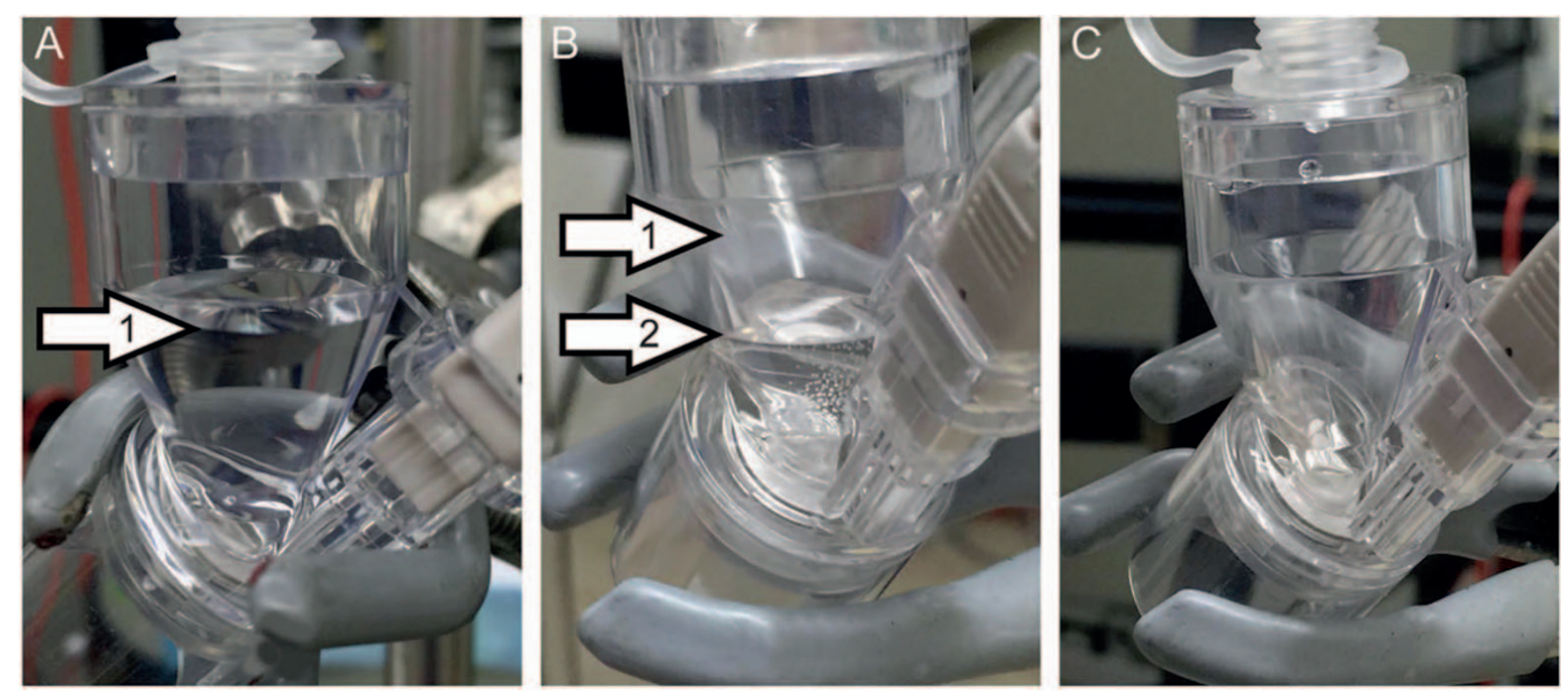

Fonte: Gowda, $2017^{10}$

Recomenda-se especial atenção na conexão do cânister (tubo pressurizado com a medicação) ao adaptador, devido o risco de vazamento de ar e contaminação. Há possibilidade de aumento do espaço morto instrumental, fazendo-se necessários ajustes ventilatórios compensatórios. Uma avaliação minuciosa da mecanica respiratória deve ser feita após administração do dispositivo escolhido.

\section{Cuidados ao administrar a aerossolterapia em pacientes sob ventilação mecânica}

As seguintes precauções devem ser adotadas durante a aplicação de aerrossolterapia em pacientes sob $\mathrm{VM}^{1,3,8}$ :

1. Realizar a remoção de secreções do tubo endotraqueal;

2. Assegurar volume corrente adequado ao paciente;

3. Se possível, reduzir o fluxo inspiratório ( $\leq 60 \mathrm{Lpm})$;

4. Agitar o MDI com objetivo de dispersar o propelente e manter a droga em suspensão;

5. Esperar cerca de 20 segundos entre as aplicações de cada dose.

\section{Aerossolterapia em pacientes não intubados}

O uso de agentes nebulizadores para o tratamento de pacientes não intubados com COVID-19 não é recomendado. Assim como na VM invasiva, o uso de nebulizadores aumenta o risco de geração de aerossol e transmissão da infecção para profissionais de saúde, pacientes e circulantes. Sempre que possível, é recomendado o uso de MDI acoplados a espaçadores. 


\section{Considerações Finais}

A terapia inalatória é frequentemente utilizada em pacientes internados nas UTIs. Pela alta demanda e por suas particularidades, a ASSOBRAFIR recomenda que o adequado uso dessa via de administração siga rigorosamente as formalidades descritas nesse documento, baseado na literatura científica atualizada, adotando-se os cuidados necessários, com o objetivo de se atingir os efeitos clínicos pretendidos e de se garantir a proteção do profissional, paciente e circulantes.

\section{Referências}

1. Dhanani J, Fraser JF, Chan HK, Rello J, Cohen J, Roberts JA. Fundamentals of aerosol therapy in critical care. Crit Care. 2016 Oct 7;20(1):269. doi: 10.1186/s13054-016-1448-5.

2. Alhazzani W, Møller MH, Arabi YM, Loeb M, Gong MN, Fan E, et al. Surving Sepsis Campaign: guidelines on the management of critically ill adults with Coronavirus Disease 2019 (COVID-19). Intensive Care Med. 2020 May;46(5):854-887. doi: 10.1007/s00134-020-06022-5. Epub 2020 Mar 28.

3. Ari A. Aerosol therapy in pulmonary critical care. Respir Care. 2015 Jun;60(6):858-74; discussion 874-9. doi: $10.4187 /$ respcare.03790.

4. Hui DS, Chan MT, Chow B. Aerosol dispersion during various respiratory therapies: a risk assessment model of nosocomial infection to health care workers. Hong Kong Med J. 2014 Aug;20 Suppl 4:9-13.

5. Whittle JS, Pavlov I, Sacchetti AD, Atwood C, Rosenberg MS. Respiratory Support for Adult Patients with COVID-19. J Am Coll Emerg Physicians Open. 2020 Apr 13;10.1002/emp2.12071. doi: 10.1002/emp2.12071. Online ahead of print.

6. Ferioli M, Cisternino C, Leo V, Pisani L, Palange P, Nava S. Protecting healthcare workers from SARS-CoV-2 infection: practical indications. Eur Respir Rev. 2020 Apr 3;29(155):200068. doi: 10.1183/16000617.0068-2020. Print 2020 Mar 31.

7. Thomas P, Baldwin C, Bissett B, Boden I, Gosselink R, Granger CL, et al. Physiotherapy management for COVID-19 in the acute hospital setting: clinical practice recommendations. J Physiother. 2020 Apr;66(2):73-82. doi: 10.1016/j.jphys.2020.03.011. Epub 2020 Mar 30.

8. Jeria RA, Valverde DW, Gajardo FB, Shaaf MIS, González PF. Guía de Recomendaciones de Armado y Uso de filtros en Ventilación Mecánica COVID19. Santiago: Sociedad Chilena de Medicina Intensivista; [2020]. Available from: https://www.medicina-intensiva.cl/site/covid/armado filtros.pdf.

9. Dhand R. Inhalation Therapy With Metered-Dose Inhalers and Dry Powder Inhalers in Mechanically Ventilated Patients. Respir Care. 2005 Oct;50(10):1331-4; discussion 1344-5.

10. Gowda AA, Cuccia AD, Smaldone GC. Reliability of vibrating mesh tecnology. Respir Care. 2017 Jan;62(1):65-69. doi: 10.4187/respcare.04702. Epub 2016 Nov 15.

11. Respiratory Therapeutics Group. Closed Suction System Double Swivel 12 Fr. Los Angeles: Respiratory Therapeutics Group; 2017 [cited 2020 Apr 22]. Available from: https://rtghealth.com/ product/closed-suction-system-double-swivel-12-fr/

Submissão em: 05/06/2020

Aceite em: 15/07/2020 